\title{
Introduction to Brain Tumor Immunology
}

\author{
KeVIN O. LiLleheI, M.D.
}

The "decade of the brain" has been a period of significant advancement in our understanding of the complex interactions occurring within the brain, the interrelated function of various neurotransmitters, and the underlying molecular biological function of diseases of the brain. In this period, our understanding of the immunological aspects of disease has also made great strides, particularly in the relationship of the immune system to cancer. Unfortunately, this insight has had little impact on our ability to treat malignant brain tumors. At present, we continue to rely on a combination of surgery, radiotherapy, and chemotherapy, and the results are universally poor. This application of our newly gained knowledge of immunology to the treatment of malignant brain tumors is an area of intense interest that holds the promise of revolutionizing the field of neurooncology.

This edition of Neurosurgical Focus is dedicated to introducing the neurosurgeon to the concepts of immunotherapy as they relate to the treatment of patients with brain tumors. Our goals are to acquaint the neurosurgeon with current immunological jargon, provide an understanding of the immune system as it relates to the central nervous system, and introduce the neurosurgeon to current strategies for manipulation of the immune system to treat intracranial tumors. Historically immunology has been a field foreign to the practicing neurosurgeon, but it is a field that is becoming increasingly important in many areas of medical care.

This issue is divided into three sections. Section I provides an overview of immunology as it pertains to the central nervous system and serves to update the neurosurgeon on current principles of immunology and immunotherapy. Section II presents a number of basic science and animal studies in which investigators explore tumor-induced immunosuppression and novel strategies for manipulation of the immune system to induce tumor regression. Section III presents the results of two clinical trials in which immunotherapy approaches are applied to the treatment of recurrent malignant gliomas.

Brain tumor immunotherapy is an exciting new area of research and one in which neurosurgeons have taken a prominent role. Despite the ability to induce a tumor-specific systemic immune response, the challenge to eradicate intracranial gliomas remains. Whether this is because of difficulties in generating an effective intracranial immune response, due to the brain's partial immune-privileged status, or the phenomenon of tumor-induced immunosupression remains unclear. Our knowledge, however, increases with each new study, bringing the potential of brain tumor immunotherapy closer to reality.

We would like to thank all of the authors for their contributions to this edition of Neurosurgical Focus and we hope that the information shared in this journal will help stimulate others to take up the challenge. We would like specifically to thank Dr. William C. Broaddus and Dr. John Sampson for their help in putting together this edition. 